\title{
Efficacy, safety and tolerability of linezolid for the treatment of XDR-TB: a study in China
}

\author{
To the Editor:
}

Heterogeneity amongst cohorts of patients treated for drug-resistant tuberculosis (TB) make assessing the efficacy of new drugs and regimens unreliable without randomisation [1]. We warmly welcome the randomised controlled trial (RCT) "Efficacy, safety and tolerability of linezolid for the treatment of XDR-TB: a study in China" presented by TANG et al. [2]. Linezolid is now included on the WHO list of essential medicines [3] and falling prices will improve access. That TANG et al. [2] report a 35.3\% absolute reduction in the risk of a poor outcome by adding linezolid to an optimised background regimen argues in favour of wider use. Linezolid remains a WHO "Group 5" drug, classifying it as an "anti-TB drug with limited data on efficacy and/or long term safety in the treatment of [drug-resistant]-TB" [4]. The limited data on the efficacy of drugs in this class has recently been reviewed [5]. These WHO classifications may shortly be revised and the exciting results from this study have the potential to alter guidance regarding the treatment of extensively drug-resistant (XDR) TB. We note the proposal to re-classify linezolid into Group 3 in an editorial in the October edition of the European Respiratory Journal [6]. It is with this in mind that we seek some clarifications.

The journal rightly requires that reports of randomised controlled trials conform to the CONSORT guidelines [7]. We were therefore concerned to note that limited details were presented regarding the randomisation process and steps taken to ensure adequate allocation concealment. Details on the target sample-size calculation were also absent. The authors do not state whether the trial was registered in a clinical trial registry as per journal guidelines.

A recent systematic review and meta-analysis by ZHANG et al. [8] highlighted linezolid's significant side-effect profile. The data presented by TANG et al. [2] on rates of nausea and on the timing of myelosuppression and neuropathy will be of great interest to clinicians and patients. The manuscript states that "Most adverse events resolved after reducing the dosage of linezolid or temporarily discontinuing linezolid, and only two patients were permanently discontinued from using the drug because of severe anaemia." Details of symptom severity and the number of patients left with cytopaenias or significant residual neuropathy would be hugely valuable.

Figure 2 suggests that around a quarter of patients in the control group achieved sputum culture-conversion in the first 3 months of the study, despite all having been sputum smear-positive for the preceding 12 months. This suggests that new agents other than linezolid were introduced in both arms at enrolment. It would be helpful to know whether other drugs that patients had not used previously were started when the patients were enrolled in the trial. In particular, many patients were reported to have been treated with clofazimine, an agent shown by the authors to be useful in the treatment of MDR-TB [9]. It would also be relevant to know whether the background regimen was determined prior to randomization, and whether there was any imbalance in newly-introduced drugs between the trial arms, both at enrolment and subsequently.

Finally, early relapse following treatment is common [10]. Data on relapse-free survival from the other completed randomised controlled trial involving linezolid has been valuable in understanding the role of this drug [11]. Relapse-free survival is a more robust end-point and follow up for at least 1 year should be routine in randomised evaluations of TB treatment regimens. We hope this will be considered in future trial designs.

If the authors could provide these additional details, it would greatly inform our understanding of the efficacy and place of linezolid in XDR regimens. This information would also be valuable to those who will shortly be drafting new international recommendations for DR-TB as well as for clinicians and programmes providing XDR-TB care. We congratulate the authors on an important study and look forward to their response. may impact new guidelines http://ow.ly/Z1dX5 
Catherine Berry ${ }^{1}$, Tom A. Yates ${ }^{2}$, James A. Seddon ${ }^{1,3}$, Patrick P. J. Phillips ${ }^{4}$ and Philipp du Cros ${ }^{1}$ on behalf of the North London TB Journal Club

${ }^{1}$ Manson Unit, Médecins Sans Frontières (MSF), London, UK. ${ }^{2}$ Research Department of Infection and Population Health, University College London, London, UK. ${ }^{3}$ Department of Paediatrics, Imperial College London, London, UK.

${ }^{4} \mathrm{MRC}$ Clinical Trials Unit, University College London, London, UK.

Correspondence: Catherine Berry, Manson Unit, Médecins Sans Frontières (MSF), Chancery Exchange, 10 Furnival St, EC4A 1AB, London, UK. E-mail: catherine.berry@london.msf.org

Received: Oct 62015 | Accepted after revision: Dec 202015

The views expressed in the submitted article are our own and not an official position of any of our institutions.

Conflict of interest: Disclosures can be found alongside the online version of this article at erj.ersjournals.com

\section{References}

1 Brigden G, Nyang'wa B-T, du Cros $\mathrm{P}$, et al. Principles for designing future regimens for multidrug-resistant tuberculosis. Bull World Health Organ 2014; 92: 68-74.

2 Tang S, Yao L, Hao X, et al. Efficacy, safety and tolerability of linezolid for the treatment of XDR-TB: a study in China. Eur Respir J 2015; 45: 161-170.

3 World Health Organization. WHO Model List of Essential Medicines. 2015. www.who.int/medicines/publications/ essentialmedicines/en/ Date last updated: August, 2015. Date last accessed: October, 2015.

4 World Health Organization. Companion handbook to the WHO guidelines for the programmatic management of drug-resistant tuberculosis. 2014. http://apps.who.int/iris/bitstream/10665/130918/1/9789241548809_eng.pdf? ua $=1 \&$ ua $=1$ Date last accessed: October 2, 2015.

5 Winters N, Butler-Laporte G, Menzies D. Efficacy and safety of World Health Organization group 5 drugs for multidrug-resistant tuberculosis treatment. Eur Respir J 2015; 46: 1461-1470.

6 Caminero JA, Scardigli A. Classification of antituberculosis drugs: a new proposal based on the most recent evidence. Eur Respir J 2015; 46: 887-893.

7 Moher D, Hopewell S, Schulz KF, et al. CONSORT 2010 Explanation and Elaboration: updated guidelines for reporting parallel group randomised trials. BMJ 2010; 340.

8 Zhang X, Falagas ME, Vardakas KZ, et al. Systematic review and meta-analysis of the efficacy and safety of therapy with linezolid containing regimens in the treatment of multidrug-resistant and extensively drug-resistant tuberculosis. J Thorac Dis 2015; 7: 603-615.

9 Tang S, Yao L, Hao X, et al. Clofazimine for the treatment of multidrug-resistant tuberculosis: prospective, multicenter, randomized controlled study in China. Clin Infect Dis 2015; 60: 1361-1367.

10 Marx FM, Dunbar R, Enarson DA, et al. The temporal dynamics of relapse and reinfection tuberculosis after successful treatment: a retrospective cohort study. Clin Infect Dis 2014; 58: 1676-1683.

11 Lee M, Song T, Kim Y, et al. Linezolid for XDR-TB: final study outcomes. N Engl J Med 2015; 373: 290-291.

\section{Benefit of treatment of latent tuberculosis infection in individual patients}

To the Editor:

In their recent article, Dobler et al. [1] described a decision aid that estimates a net benefit of treatment for latent tuberculosis infection (LTBI) in a low tuberculosis incidence environment. While the aid probably confers some clinical utility as described, its dynamic robustness could be strengthened in some important respects. In a series of previous articles [2-4] using primary longitudinal data from an urban American tuberculosis clinic that would be likely to benefit from use in some form of these authors' decision aid, I showed empirically a number of outcomes relevant to LTBI treatment in a low-incidence, mostly immigrant setting. First, nonadherent patients are likely to experience more adverse and expensive LTBI treatment outcomes (had they completed therapy) [2, 4], and potentially, greater likelihood of TB re-activation [3]; second, a transitional approach to treatment improves the net benefit received over any isoniazid $(\mathrm{H})$ monotherapy (6 months $(6 \mathrm{H})$ or $9 \mathrm{H})$ [4]; and third, ethnic heterogeneity in an immigrant clinic population may provide clinicians with guidance on tolerability to isoniazid [4]. 